\title{
Clinical MR Neuroimaging: Physiological and Functional Techniques
}

\author{
J.H. Gillard, A.D. Waldman, and P.B. Barker, eds. \\ New York, NY: Cambridge University Press, 2010, 891 pages, $\$ 295$
}

This book is a second edition of Clinical MR Neuroimaging: Diffusion, Perfusion and Spectroscopy, published 5 y ago, with the subtitle changed to reflect the broadening of the subject material that has occurred with the continuous evolution of physiologic and functional neurologic MRI techniques.

The last several decades have seen remarkable advances in the clinical neurosciences, with some of the greatest achievements being related to neuroimaging. Although CT generally shows only morphology - and that at a relatively low contrast and spatial resolution-it allows the traditional lesion-deficit methodology to be applied to living subjects. The need for functional and molecular imaging of the brain was met by the combination of PET and metabolic radiotracers. The development of PET was followed relatively quickly by MRI. Conventional MRI relies on electromagnetic signals emitted by water molecules of relatively stationary tissue. MRI allows the detailed depiction of brain anatomy and pathology with unprecedented spatial resolution and soft-tissue contrast. It is also relatively safe and completely noninvasive. However, the diagnostic accuracy with which structural MRI alone can define the wide range of neurologic diseases is limited. The last decade has seen the development of physiologic MRI techniques, whereby information concerning tissue function is obtained. These techniques include diffusion, perfusion, and MR spectroscopy, which provide information on tissue ultrastructure, blood flow, and biochemistry, respectively. These often provide important surrogate markers of disease pathophysiology or therapeutic response. These techniques, previously available only in a research environment, are now accessible on most MRI scanner systems and can readily be incorporated into clinical imaging tests. Today, however, there is a paucity of single-volume books to support those wishing to apply physiologic imaging studies to a clinical context.

This edition has been updated not only to reflect the latest developments in diffusion- and perfusion-weighted imaging and MR spectroscopy but also to include functional MRI, permeability imaging, and susceptibility-weighted imaging. Currently, diffusion imaging, perfusion imaging, and spectroscopy are the physiologic techniques most likely to be used routinely. Functional MRI has become synonymous with studies of localized brain activation, mostly using blood oxygen level-dependent contrast, and has been used for presurgical brain mapping. Susceptibility-weighted imaging is exquisitely sensitive in the detection of hemorrhage or venous deoxygenation. Other advanced methods, such as magnetization transfer imaging, volumetric MRI, and MRI relaxometry, are not addressed in this edition because they are well covered in other texts and remain primarily in the research realm. They are not widely available and have yet to find a definite clinical role.

This book is organized into 9 sections with 55 chapters written by 105 authors. As in the first edition, section 1 deals with physiologic MRI techniques, as well as fundamentals and potential artifacts of MR spectroscopy and of diffusion, perfusion, and functional MRI. The other 8 sections discuss the latest physiologic and molecular MRI methodologies in detail and then illustrate their applications in cardiovascular disease, adult neoplasia, infection, inflammation and demyelination, seizure disorder, psychiatric and neurodegenerative disease, trauma, pediatrics, and the spine. Chapters are grouped according to disease and are preceded by overviews that place these methodologies in a broader clinical perspective. New sections on the use of functional MRI for presurgical planning, as well as susceptibility and contrast-based permeability, are particularly useful in clinical practice. A further 73 illustrative case studies have been added, including both new and old techniques. The 605 figures are clear and illustrative, and the 37 tables are informative and useful. References are updated, and the index is helpful.

The goal of this book is to address the appropriate clinical application and interpretation of diffusion and perfusion MRI and MR spectroscopy and thus create a widely applicable reference work for those techniques. Another goal is to optimize the use of these techniques to increase the diagnostic accuracy of MRI and thereby improve the management of individual patients. It is hoped that the advanced MRI techniques provide biomarkers that can be used in trials of new therapies, thus leading to better treatments and outcomes. New MRI techniques of the early 21st century foreshadow even more remarkable advances in neuroimaging but appreciate the robust functional imaging capabilities described and illustrated in this book. I am sure that the clinical value of these physiologic and molecular tools will become increasingly appreciated and helpful in the management of neurologic patients. I highly recommend this book to trainees and practitioners in the fields of radiology, nuclear medicine, neurology, neurosurgery, emergency medicine, and physical medicine.

\author{
E. Edmund Kim \\ M.D. Anderson Cancer Center \\ 1515 Holcombe Blvd., Unit 1264 \\ Houston, TX 77030 \\ E-mail: ekim@di.mdacc.tmc.edu
}

